Vierteljahrsschrift für Geschichte der Medizin und der Naturwissenschaften Revue trimestrielle d'histoire de la médecine et des sciences naturelles

Redaktion/Rédaction: Heinz Balmer, Zürich
GESNERUS

Jahrgang/Vol.38 1981

Heft / Fasc. 3/4

Verlag/Editions Sauerländer

\title{
Zur Entstehung und Entwicklung des kindlichen Hysteriebegriffes
}

Von Elisabeth Kloë und Hildburg Kindt

\section{Einleitung}

Die historische Untersuchung von Krankheitsbezeichnungen, die altersund phasenspezifische Vorstellungen beinhalten, muß von zwei grundsätzlichen Fragestellungen ausgehen: erstens von der Frage nach der Beziehung zwischen der zu untersuchenden Krankheitsbezeichnung und der betreffenden Lebensphase, zweitens von der Frage nach dem theoretischen Hintergrund, der für die Entwicklungsgeschichte eines solchen Zusammenhanges verantwortlich gemacht wird. Daraus ergibt sich zwingend die Frage nach der gegenseitigen Bedingung von phasenspezifischen Krankheitsbenennungen und den ihnen zugrundeliegenden theoretischen Konzepten.

Ausgehend von dieser Fragestellung erscheint die «kindliche Hysterie» besonders interessant, weil sich die Anwendbarkeit oder Nicht-Anwendbarkeit der traditionellen Konzepte auf das Kindesalter mit der Frage nach der Ätiologie hysterischer Zustände als auf das Engste verknüpft erweist. Die früheste Auffassung von Hysterie als somatische Erkrankung der Gebärmutter der geschlechtsreifen Frau blieb von der Antike bis ins 17. Jahrhundert weitgehend unangefochten $(9,35,53)$. Sie läßt sich in dem Gedanken, Hysterie als Genitalneurose zu begreifen $(37,41,42,46)$ bis ins 19 . Jahrhundert hinein verfolgen. Diese Tradition stand im Gegensatz zu der Entwicklung eines kindlichen Hysteriebegriffes. In der vorliegenden Arbeit soll 
diesen zwei scheinbar entgegengesetzten Hysterievorstellungen näher (35) nachgegangen werden.

\section{Von der Gebärmutterkrankheit zur funktionellen Nervenerkrankung}

\section{Konzepte der Antike (uterine Genese)}

Der Terminus «Hysterie» weist durch seine Herkunft von dem griechischen

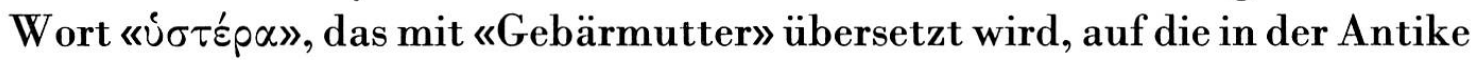
vorherrschende und bis ins 19. Jahrhundert relevante Vorstellung von der Ätiologie und dem Sitz der Krankheit Hysterie im Uterus hin. Als «Frauenkrankheit» war die Hysterie in den Schriften der Hippokratiker ein Leiden mit einer natürlichen Ursache und war gekennzeichnet durch eine Vielzahl von Symptomen, insbesondere durch Erstickungs- und Konvulsionserscheinungen, «hysterische» Organsymptome wie Lähmungen und Blindheit und vegetative Begleiterscheinungen wie Herzklopfen oder starker Schweißausbruch (Corpus Hippocraticum, 1. und 2. Buch über die Frauenkrankheiten und 32). Die Auffassung der Hysterie als Gebärmutterkrankheit, die durch das Umherwandern des Uterus im Körper (Hippokrates, 460-377 v. Chr., Platon, 428/27-347 v. Chr., Aretäus, um 150 n. Chr.) oder durch die Stauung weiblichen Samens oder menstruellen Blutes (Galen, um 150 n.Chr., Soranus, um 100 n. Chr.) verursacht wird, gründet in den Theorien der antiken Autoren letzten Endes auf sexueller Abstinenz und der Versagung der natürlichen Geschlechtsfunktionen. Dieser Vorstellung folgend glaubte man, daß Hysterie durch Heirat und Schwangerschaft geheilt werden könne (27; Teil 24, S. 34). Das Hysteriekonzept der Antike beinhaltet bereits die wesentlichen Voraussetzungen für die Entwicklung der problematischen Beziehung des Hysteriebegriffes zum Kindesalter: die Verbindung von Hysterie mit Sexualität und das ausschließliche Vorkommen der Hysterie bei Frauen im geschlechtsreifen Alter. Die besondere Problematik liegt darin, daß die Gedankenverknüpfung von Hysterie mit der Sexualität des weiblichen Geschlechts in medizinischen und außermedizinischen (19) Betrachtungsweisen bis in das 19. Jahrhundert erhalten blieb, obwohl ein ursächlicher Zusammenhang zwischen dem Uterus und den vielgestaltigen hysterischen Symptomen spätestens seit dem Ende der Antike in Frage gestellt wurde.

Die Beobachtung hysteriformer Krankheitszustände auch bei Männern 
hätte schon sehr früh die Möglichkeit angeboten, den Uterus als ätiologische Quelle hysterischer Zustände auszuschalten. Bei Galen (23; Bd. VIII, S. 417, 418) und Aretäus (2; S. 124, 125), die solche Fälle beobachteten, ist aber nur von einer «ähnlichen Form der Krankheit» bei Männern die Rede, von der man die echte Hysterie als Gebärmutterkrankheit durchaus unterscheiden könne. Bei Galen fällt auf, daß als Ursache für Hysterie und für jene Frauen und Männer gleichermaßen betreffenden hysterieähnlichen Krankheiten die sexuelle Abstinenz genannt wird. Beiden Krankheitsformen war zumindest eine Ursache gemeinsam, die im Sinne einer somatischen Reaktion auf die Versagung einer für den Organismus notwendigen Funktion verstanden wurde. Die antiken Ärzte unterschieden medizinisch eindeutig zwischen den geschlechtlichen Funktionen als einem natürlichen, rein physischen Bedürfnis des Menschen einerseits und ihrem psychischen Anteil andererseits, der in der Hysterielehre der Antike aber noch keine Rolle spielte (53; S. 42, 43). Das Vorkommen von Hysterie bei Männern wurde in der zeitgenössischen und nachfolgenden Literatur jedoch ignoriert und erst eineinhalb Jahrtausende später von Charles Lepois (= Carolus Piso, 1563-1633) und Thomas Willis (1621-1675) wiederentdeckt und noch einmal ignoriert, bis diese Beobachtung in der Mitte des 19. Jahrhunderts als wesentliches Element in der Hysteriediskussion wieder auftauchte.

\section{Hysterie im 17. Jahrhundert (cerebrale Genese)}

Lepois verlegte in seinem 1618 erschienenen Werk: «Selectiorum observationum et consiliorum liber singularis» die Ursache der Hysterie vom Uterus in den Kopf. Die hysterischen Symptome, die bisher dem Uterus, dem Magen und anderen inneren Organen zugeschrieben wurden, kämen vom Kopf, und zwar sei dieser nicht «sympathisch», sondern «idiopathisch» erkrankt (43; S. 144). Das Symptom der Kopfschmerzen, die sowohl bei hysterischen Frauen als auch Männern vorkämen, ist für Lepois ein Beweis dafür, daß Hysterie beide Geschlechter gleichermaßen betreffend (43; S. 181) durch den Kopf verursacht werde. Dementsprechend findet sich bei Lepois die erste Erwähnung, daß Hysterie auch schon im kindlichen Alter auftreten könne (43; S. 13).

Fünfzig Jahre später berichtet auch Thomas Willis in seinem Werk «... de morbis convulsivis ...» (1668) vom Ursprung und Sitz der Hysterie im Gehirn. Willis lehnt die uterine Genese der Hysterie aufgrund seiner 
pathologisch-anatomischen Studien ab, da ein «Aufsteigen» des Uterus infolge der anatomischen Verhältnisse unmöglich sei und er bei der Autopsie von Hysterischen keine Schäden der Gebärmutter gefunden habe. Hingegen seien die Nervenursprünge im hinteren Teil des Kopfes durch eine Flüssigkeit überschwemmt gewesen. Als Bestätigung für seine Theorie führt er an, daß Hysterie in jedem Alter, auch bei Mädchen vor der Pubertät, zu beobachten sei (54; S. 161). Auch Thomas Sydenham (1624-1689) hält 1681 eine angeborene oder erworbene Schwäche der «Lebensgeister» für die eigentliche Ursache der Hysterie (52; S. 95).

Die Feststellung, daß Hysterie schon im Kindesalter vorkommt, diente Lepois und Willis als Beweis für ihre Annahme, daß Hysterie ein Kopfleiden sei. Beide Autoren können die kindliche Hysterie aber noch nicht als eigenständige Krankheitsform begreifen. Lepois' Theorie der Hysterie als idiopathisches Kopfleiden scheint aber eine Vorläuferfunktion für die spätere Auffassung der Hysterie als einer nervös-funktionellen Erkrankung zu haben. Willis' und Sydenhams Gedanken weisen dagegen in Anlehnung an die Theorien der «spiritus animales», von denen man glaubte, daß sie an den Nervenursprüngen lokalisiert seien und durch Gemütsbewegungen geschwächt werden könnten (54; S. 165), auf eine mehr psychologisch interpretierbare Erklärung, was Hysterie sei, hin.

\section{Hysterie im Rahmen des organischen Neurosekonzeptes}

Im 18. und in der ersten Hälfte des 19. Jahrhunderts erfuhr die Diskussion über die Ätiologie und Genese der Hysterie eine neue Wende. In den Vordergrund trat nun die Frage, ob die Hysterie eine «Neurose» sei*.

Bei genauerer Untersuchung der Hysterietheorien des 18. und 19. Jahrhunderts zeigte sich, daß humoralpathologische Denkweisen, die in den Hysteriekonzepten des 16. und 17. Jahrhunderts von wesentlicher Bedeutung waren, und die seit dem 17.Jahrhundert eindeutig nachweisbare Annahme einer nervösen Ätiologie, die schließlich zur Subsumierung von Hysterie unter die «Neurosen» führte, verschiedene Konsequenzen gehabt

* Zur Geschichte des Neurosebegriffes vgl. Binder (1962), Fischer-Homberger (1970a; S. 78-81 und 113-122) und Lopez Piñero (1963). «Die Neurose des 19. Jahrhunderts war nicht ... eine psychogene Krankheit, sondern entweder eine Nervenkrankheit oder eine Krankheit mit unbekanntem oder unauffindbarem Substrat» $(20 ;$ S. 78). 
hatten. Im Rahmen humoralpathologischer Denkweisen hatten sich entweder die «vapeurs», $(35 ; \mathrm{S} .31,32)$, toxische Dämpfe, die vom Uterus aufsteigen und die verschiedenen Organe, vor allem das Nervensystem, schädigen, oder aber die Körpersäfte (Blut oder eine vielfach angenommene Nervenflüssigkeit) zur Erklärung der hysterischen Symptome angeboten. Andererseits - und dies läßt sich als Vorstufe zu einer nervösen Ätiologie betrachten - war eine enge Verbindung zwischen den Nerven des Uterus und denen anderer Organe (gedacht als sympathische Verbindung zwischen den inneren Organen und dem Rückenmark oder Gehirn) für möglich gehalten worden. Vielfach waren auch beide Denkweisen vereinigt worden.

Die Unsicherheit in der Erklärung der hysterischen Symptome führte im Laufe des 18. Jahrhunderts zunächst zu einer zunehmenden Auflösung der Hysterie als Krankheit $(9,20,35,53)$. Ähnlich wie der Begriff «vapeurs» erfuhr der Terminus «Hysterie» einen Bedeutungswandel von einem mehr ätiologisch begründbaren zu einem nur noch symptomatologisch faßbaren Begriff. Die Klassifizierung von Hysterie als «affection vaporeuse» (45) beinhaltete gleichermaßen die von alters her gültige Auffassung, daß Hysterie ein Krampfleiden sei, als auch die Assoziation mit bestimmten Eigenschaften der weiblichen Psyche bei Hysterie (32; S. 1566, 1567; 45).

William Cullen (1712-1790) gebrauchte offenbar als erster den Begriff «Neurose» (1777). Er zählt die Hysterie in seiner «Nosologie» zu den «krampfichten Krankheiten» (14; Erster Teil, S. 248 und 329-332), und gibt dem Uterus noch einen beachtlichen Anteil an der Pathogenese der Hysterie: «... die Anfälle des hysterischen Uebels nehmen mit einer convulsivischen und spasmodischen Bewegung des Canals der ersten Wege ihren Anfang, welche krampfhafte Zufälle hernach dem Gehirn und einem großen Theil des Nervensystems mitgetheilt werden» (15; Bd.3, S.596). In ähnlicher Weise wie Cullen findet auch Joseph Lieutaud (1703-1780) einen Kompromiß zwischen Genital- und Nervenfunktionen (39; Bd.3, S. 176, 177). Philippe Pinel (1745-1826), Jean-Baptiste Louyer-Villermay (1776-1837), MarcHector Landouzy (1812-1864) und Moritz Heinrich Romberg (1795-1873) halten die Hysterie für eine «Genitalneurose» der Frau (42, 41, 37, 46).

Den Vertretern genitaler Theorien standen jedoch in der Nachfolge von Lepois, Willis und Sydenham in der ersten Hälfte des 19. Jahrhunderts Autoren wie Joseph Raulin (1708-1784), Etienne-Jean Georget (17951828), Félix Voisin (1794-1872), Benjamin C. Brodie (1783-1862) und JeanLouis Brachet (1789-1858) als Verfechter einer rein nervösen Ätiologie entgegen. So hält Georget die Hysterie für ein Gehirnleiden, eine «cérébropa- 
thie» (24; Bd.2, S.261, 262). Einen Unterschied zwischen Hysterie und Epilepsie als den beiden klassischen Neurosen sieht er lediglich im Schweregrad der Krankheit (24; Bd.2, S.390). Diese unscharfe Abgrenzung der Hysterie von anderen Neurosen, die sich auch in den Einteilungsschemata zahlreicher anderer Autoren finden läßt (7, 14, 22, 24, 42), verhinderte vermutlich die Anerkennung von Hysterie als einer funktionellen oder organischen Störung des Gehirns oder Nervensystems. Johann-Peter Frank (1745-1821), der die Hysterie ebenfalls zur Klasse der «Nervenkrankheiten» (Neuroses) zählt (22; Bd.9, S.290, 291), berichtet über die Ratlosigkeit der Hysterieforschung seiner Zeit, die bisher weder einen «krankhaften Zustand an den Geschlechtstheilen» noch eine «Ursache ... in dem Gehirn oder den Nerven, die von anderen Theilen zu der Gebärmutter kommen ...», gefunden habe (22; Bd. 8, S.265).

Die gleiche diagnostische Zuordnungsproblematik gilt für die Beurteilung der wenigen Fälle kindlicher Hysterie. Abgesehen von Einzelbeobachtungen, die keinen nachhaltigen Eingang in die ohnehin spärliche pädiatrische und kinderpsychiatrische Literatur fanden, wurde im 18. und in der ersten Hälfte des 19. Jahrhunderts keine kindliche Hysterie diagnostiziert. Die Bedeutung der Einzelbeobachtungen liegt auch weniger in der Abgrenzung kinderpsychiatrischer Krankheitsbilder, als vielmehr darin, den Gedanken, daß Hysterie eine Frauenkrankheit sei, in Frage zu stellen, um damit den Weg zu einer Umorientierung in der Auffassung der Hysterie als Psychoneurose freizumachen.

In der Diskussion zwischen den Verfechtern einer mehr somatischgenitalen und einer funktionell-nervösen Hysterietheorie war die Frage nach Alter und Geschlecht der Kranken von wesentlicher Bedeutung. Die Bejahung oder Verneinung des Vorkommens einer kindlichen Hysterie wies entweder in die eine oder die andere Richtung. Während für andere «Neurosen» wie zum Beispiel für Epilepsie eine dem Erwachsenenalter analoge Betrachtungsweise auch im Kindesalter möglich war, wurden klinische Beobachtungen kindlicher Hysterie insbesondere für die Vertreter genitaler Theorien zum ungeklärten wissenschaftlichen Problem. Sie betrachteten Fälle kindlicher Hysterie daher als «Ausnahmen von der Regel» oder versuchten sie durch die Annahme einer zu frühen Reifung der Genitalorgane oder den zu frühen Eintritt der Pubertät zu erklären oder aber waren bemüht, die bei anderen Autoren beschriebenen Fälle kindlicher Hysterie als Fehldiagnosen zu entlarven. In diesem Sinne gibt auch Cullen zu, daß die Hysterie «zuweilen vor ... den Jahren der Mannbarkeit ...» 
auftreten könne, relativiert seine Aussage dann aber sofort, wenn er bemerkt, daß die «Zufälle des hysterischen Uebels ... zuweilen bey jungen noch nicht mannbaren Mädchen mit dem Veitstanz verknüpft .... seien (15; S. 592). Louyer-Villermay spricht hingegen von einer weniger stark ausgeprägten Sonderform der Hysterie bei Mädchen vor der Pubertät, die er als «hystéricisme» bezeichnete $(41 ;$ S. 242, 243). Landouzy sieht die Disposition für die Hysterie in der Pubertät in funktionellen Störungen in der Entwicklung der Genitalorgane (37; S. 183, 184). Dagegen waren den oben genannten Verfechtern einer zentralnervösen Ätiologie wie Raulin (1758), Georget (1821) und Brachet (1849) Fälle kindlicher Hysterie als Beweis für ihre Theorien willkommen (vgl. 45; S.45-49; 13; S.14; 4; S. 12). Diese mehr theoretisch interessante Auseinandersetzung darf nicht darüber hinwegsehen lassen, daß die ersten Fallschilderungen von kindlicher Hysterie, die allerdings noch keine Ansätze für eine kinderspezifische Betrachtungsweise enthalten, die Entstehung eines kindlichen Hysteriebegriffes in der zweiten Hälfte des 19. Jahrhunderts vorbereiten. Unter anderen wiesen später Paul Briquet (1796-1882), Arthur Clopatt (geb. 1858) und Paul Bézy (geb. 1855) auf die Wichtigkeit dieser Fallschilderungen hin (8; S. 52-54; 13; «Appendice» III-VII; 4; S.9-12).

Die funktionell-nervösen Hysterietheorien einschließlich des organischen Neurosekonzeptes erwiesen sich aber als letztlich nicht geeignet, um die genitalen Theorien zu verdrängen. Die hier aufgezeigten Schwierigkeiten in der Ätiologiediskussion der Erwachsenen-Hysterie stehen in engem Zusammenhang mit der Einengung des medizinischen Ursachenbegriffes des 19. Jahrhunderts auf pathologisch-anatomisch nachweisbare Läsionen und naturwissenschaftlich beweisbare Fakten.

\section{Zur Entstehungssituation eines eigenständigen kindlichen Hysteriebegriffes}

\section{Symptomatologische Zuordnungsversuche kindlicher Hysterien}

Wir haben versucht zu zeigen, daß die Pädiatrie mit einem Hysteriebegriff konfrontiert wurde, der infolge seiner - längst fragwürdig gewordenen theoretischen Implikationen eine fraglose Übertragung von Begriff und Inhalt der Hysterie auf das Kindesalter nicht zuließ. Im letzten Viertel des 18. Jahrhunderts wird der klinische Blick zunehmend in Richtung der 
nervösen Störungen des Kindesalters gelenkt. Zunächst interessieren mehr die kindlichen Anfallsleiden, was sich im Zusammenhang mit der vorwiegend neuralpathologischen Ausrichtung der medizinischen Krankheitssysteme des 18. Jahrhunderts erklären läßt (49). Neben der zunehmenden Beachtung, die kindliche Neurosen wie Chorea, Epilepsie und Eclampsie fanden, die als Krampfleiden in enger ätiologischer und symptomatologischer Beziehung zur Hysterie gesehen wurden, mußten weitere nervöse Störungen des Kindesalters, wie zum Beispiel allgemeine Convulsionen und Spasmus glottidis und die sogenannte idiopathische Lähmung abgegrenzt werden. Vor allem die letztgenannten Leiden boten aber eher den Charakter von Symptomen als den von einheitlichen Krankheitsbildern und standen insofern dem Zufluß weiterer ungeklärter Symptome in besonderem Maße offen. Auffällig ist, daß die aufgezählten einzelnen «Krankheiten» oder Symptome wichtigen Einzelsymptomen der Hysterie entsprechen. Die Durchsicht der pädiatrischen Literatur des ausgehenden 18. und der ersten Hälfte des 19. Jahrhunderts läßt erkennen, daß diese Krankheitszeichen im klinischen Umgang mit dem beobachteten, aber nicht anerkannten Phänomen einer «kindlichen Hysterie» - je nach der vorherrschenden Symptomatik - die Funktion von Ersatzdiagnosen erhielten (35; S.48-63). Dieses Vorgehen entsprach der «zunächst vorherrschenden Tendenz, die Kinderkrankheiten allgemein aus der nur wenig differenzierenden Gegenüberstellung zu den Krankheiten des Erwachsenenalters heraus zu betrachten», die «gleichermaßen die Einreihung kindlicher psychischer Auffälligkeiten entweder in die sich zunehmend somatisch-naturwissenschaftlich ausrichtende Pädiatrie oder in die Erwachsenen-Psychiatrie beinhaltete» (34; S.47). Andererseits läßt sich schon seit der Antike die Vorstellung von einer besonderen Affinität der Krampfleiden zum Kindesalter nachweisen (28; S. 28, 29, 49). Indem man in der zweiten Hälfte des 18. Jahrhunderts unter dem Einfluß der neural-pathologischen Theorien eine naturgegebene besondere Irritabilität des kindlichen Nervensystems für die Entstehung der Krampfleiden verantwortlich machte (49), hatte man für die dem Kindesalter als spezifisch zuerkannte Gruppe von Störungen auch eine spezifische Ätiologie gefunden. Sie war allerdings mit dem Mangel behaftet, daß sie für alle Formen der Kinderkrämpfe gleichermaßen in Anspruch genommen wurde. Dies hatte eine unscharfe Abgrenzung der einzelnen Formen untereinander zur Folge.

Bei der diagnostischen Zuordnung der beobachteten Fälle «kindlicher Hysterie» zu den oben genannten kindlichen Nervenleiden lassen sich schon 
frühzeitig Ansätze zu einer mehr psychologischen Betrachtungsweise des hysterischen Kindes nachweisen. So wurden zum Beispiel für die Entstehung der Chorea, die als «kindliche Form der Hysterie» bezeichnet wurde (Johann Ludwig Choulant, 1791-1861, und Eugène Bouchut, 1818-1891) (6; S. 183), unter anderem die besonders starke und nachhaltige Beeindruckbarkeit der Kinder durch Sinneseindrücke, die kindliche Suggestibilität und Phantasie und die Tendenz der Kinder zur Nachahmung verantwortlich gemacht. Bouchut sieht als ursächliches Moment der Chorea eine «gewisse angeborne nervöse Constitution oder erworbene Anlage, die später auch gern Ursache für die Hysterie wird ...» (6; S. 183). Jean-Baptiste-Timothée Baumès (1756-1828) beobachtet bei der Chorea «die unvernünftigen Gemüthsbewegungen, welche man beym hysterischen Uebel wahrnimmt» (3; S. 377). Die Patienten haben bei der Chorea «eine unüberwindliche Neigung zur Melancholie, und die Mädgen bekommen oft hysterische Zufälle ...». «Dies sind ... Erscheinungen, welche dem St. Veitstanz, der Melancholie und dem hysterischen Zufalle eigen sind ...» (3; S.380, 381). Carl Gerhardt (1833-1902) weist 1861 darauf hin, daß die Bedeutung der Imitation bei der Chorea «mindestens ebensogross sei, als bei Hysterie und Epilepsie ...» und «sowohl ätiologisch ... als auch therapeutisch alle Beachtung» verdiene (25; S.437). Auch Johann Christian Gottfried Jörg (1779-1856) warnt in seinen Empfehlungen für das «moralische Benehmen» gegenüber an Chorea erkrankten Kindern, insbesondere solchen Kranken, «deren Befinden dem hysterischen Zustande nahe steht ...», vor «falscher Theilnahme», durch die sich die Kranken «wohl auch wichtiger erscheinen und daher mit Absicht die krankhafte Stimmung nähren» (31; S. 851).

Im Sinne und Verständnis des Sensualismus wurden die durch psychogene Mechanismen wie Schreck, Angst, Nachahmung, Masturbation, Erziehungsfehler oder geistige Überanstrengung entstandenen kindlichen Krämpfe anderen kindlichen Anfallsleiden wie zum Beispiel der Epilepsie, der Eclampsie oder den allgemeinen Convulsionen zugeordnet. Bouchut ist der Ansicht, daß es sich beispielsweise «mit der Eclampsie ... bei manchen sehr empfindlichen Kindern ähnlich, wie mit den Nervenerscheinungen der Hysterischen» verhält. «Die unbedeutendsten Eindrücke prägen sich ihnen tief ein. Solche Kinder äussern auch schon sehr früh ihre Einfälle und ihre Launen, die Umgebung muss ihnen gewähren, was sie wollen.» Er beobachtete auch, daß Kinder von hysterischen Müttern häufiger von Convulsionen befallen werden. Im Gefolge der Convulsionen können Lähmungen auftreten «wie ... bei Hysterischen ...» (6; S. 199, 201, 207). Diese Beispiele 
verdeutlichen, daß es nicht das Vorkommen hysterischer Zustände beim Kind ist, das eine eigenständige kindliche Hysterie möglich macht. Vielmehr muß die Erkenntnis spezifisch kindlicher Eigenschaften und Schädigungen, die gleichzeitig als ätiologische Matrix für kindliche psychische Auffälligkeiten dienen, als wichtigster Faktor für die Entwicklung einer Hysterie im Kindesalter angesehen werden.

Die beschriebenen Zuordnungsversuche kindlicher Hysterie im 18. und in der ersten Hälfte des 19. Jahrhunderts werden nicht erst für den historischen Beobachter auffällig, sondern gewinnen in der zweiten Hälfte des 19. Jahrhunderts einen unmittelbaren und praktischen Einfluß auf das Bemühen der Pädiatrie und beginnenden Kinderpsychiatrie um die Konzeption eines auf das Kind anwendbaren Hysteriebegriffes. Die für die Diagnose Hysterie in Frage kommenden Fallbeschreibungen hysterischer Zustände werden nunmehr trotz ihrer teilweise unterschiedlichen Symptomatik in einem inneren Zusammenhang gesehen, weil die Einzelsymptome alle Wesensmerkmale der Hysterie enthielten. Die Erkenntnis dieses «inneren Zusammenhangs», nämlich, daß es sich bei bestimmten «neurotischen Symptomen» um in «ihrem Wesen nach ... naheverwandte Zustände» handelt, eröffnete den Weg zu einer von der klinischen Beobachtung her gestützten und praktisch anwendbaren Krankheitseinheit (26; S.916, 1008). Zahlreiche Autoren, die sich speziell mit kindlicher Hysterie beschäftigten, faßten nunmehr Krankheiten und Symptome als Formen der «hysterischen Zustände im Kindesalter» (26; S.916) auf (s. Tabelle). Die Frage nach der Ursache des inneren Zusammenhanges aller hysterischen Symptome, nach Ätiologie und Pathogenese der Hysterie tritt aber bei den pädiatrischen und kinderpsychiatrischen Autoren zunächst in den Hintergrund. Die Frage nach der «Identität der Hysterie Erwachsener mit derjenigen der Kinder ...» wird zwar von Eduard Henoch (1820-1910) gestellt, wird aber nicht weiter verfolgt, weil «wir auch über das Wesen der Hysterie Erwachsener so gut wie nichts wissen ...». Henoch kennzeichnet die Situation der beginnenden kinderpsychiatrischen Hysterieforschung folgendermaßen: «... man muß sich begnügen ..., einen Komplex der veschiedensten neurotischen Symptome, motorischer, sensibler, psychischer, ja selbst trophischer, die sich in stets wechselnden Verschlingungen miteinander kombiniren können, als den Ausdruck der Hysterie zu betrachten. Der Ausgangspunkt und der innere Zusammenhang der Erscheinungen bleibt uns dabei gänzlich unbekannt und die beliebte Annahme einer erhöhten Reflexerregbarkeit, einer «nervösen〉 Disposition ist nicht geeignet, die Lücke zu verdecken» (26; 
Tabelle: Symptomatologische Einteilungsversuche kindlicher Hysterie in der zweiten Hälfte des 19. Jahrhunderts

Faye (1875)

Lange (1875)

Seeligmüller (1881)

Henoch (1881)

Emminghaus (1887)
1. Paralytische Anfälle

2. Convulsive Anfälle

3. Chorea

4. Übertreibungssucht

5. Hysterische Arthralgien

6. Pica

1. Paralytische Anfälle

2. Erregungserscheinungen

3. Psychische Abnormitäten

1. die maniacalische Form (Chorea magna)

2. die hypnotische Form

3. die epileptische Form

4. die convulsive Form

1. Überwiegen der psychischen Symptome, vollständige oder unvollständige Pausen des Bewusstseins, Halluzinationen, Delirien, Katalepsie oder Eklipsis

2. Convulsivische Form (Stimm-, Wein- und Schreikrämpfe)

3. Chorea magna (grosser Veitstanz)

4. Sensible und trophische Störungen

1. Störungen der Sensibilität

2. Störungen der Motilität

3. Störungen der vasomotorischen Innervation

4. Störungen der secretorischen Innervation

5. Psychische Störungen die hysterische Gemüthsveränderung bzw. hysterische Gemüthsentartung (entspricht der gleichnamigen Charakterveränderung bei Erwachsenen)

Duvoisin (1889) die vorübergehenden hysterischen Psychosen (kataleptische Zustände, hysterisch-epileptische Anfälle, Somnambulismus, Chorea magna, Furor, religiöse Ekstase, schreckhaftes Delirium mit Phantasmen)

1. Einfachste Formen (nur Charakterveränderung und allgemeine Klagen)

2. Hysterie ohne Anfälle und ohne Störung des Bewusstseins

3. Convulsive Form mit mehr oder weniger ausgesprochener Bewusstseinsstörung

a) Anfälle von Hystero-Epilepsie s. epileptiformer Hysterie

b) Chorea magna s. major oder Chorée rythmique

c) Spasmus nutans

d) atypische, anfallsweise auftretende, tonische und klonische Krämpfe. 
S.916). Der Mangel eines unumstrittenen und auf das Kindesalter ohne weiteres übertragbaren ätiologischen Hysteriekonzeptes erweist sich jedoch als Chance für die Erkenntnis von Besonderheiten zumindest in Symptomatik und Verlauf hysterischer Zustände bei Kindern.

\section{Die kindliche Hysterie als erste Kinderneurose}

In der zweiten Hälfte des 19. Jahrhunderts wird die Frage nach der somatischen oder psychischen Genese von Neurosen auf der Basis einer vorwiegend materialistisch-naturwissenschaftlich orientierten Medizin neu gestellt $(1,21)$. Der Neurosebegriff des 19. Jahrhunderts hatte beinhaltet, daß ein pathologisch-anatomisches Substrat für die zugrundeliegende Störung noch nicht oder überhaupt nicht nachweisbar sei (40), und stand so im Gegensatz zu den anderen Nervenkrankheiten der somatischen Medizin. Hysterie als Neurose bedeutete in der zweiten Hälfte des 19. Jahrhunderts jedoch nicht nur ein Konglomerat von Symptomen mit ungeklärter Ätiologie, sondern war darüber hinaus ein Leiden, das infolge des unhaltbaren Zusammenhanges zwischen Genitalorganen und Nervensystem seinen «Sitz» und damit auch seine somatische Ätiologie verloren hatte. Dies schien problematischer zu sein, als von bestimmten Krankheiten den «Sitz» nicht oder «noch nicht» zu kennen. Das «Hysterische» wurde, wie Emil Kraepelin (1856-1927) 1889 treffend schrieb, zu einem «Beinamen», mit dem «gelegentlich eine ganze Reihe von psychopathischen Zuständen» belegt wurde (36; 3. Aufl., S.428, 429). Die diagnostische Unsicherheit in der Hysteriefrage und die seit dem 17. Jahrhundert auch historisch nachweisbaren Überlegungen zur Psychogenie der Hysterie (35; S. 84-100) scheinen uns die wesentlichen Voraussetzungen dafür zu sein, daß sich an dieser Störung die Problematik ausschließlich somatischer Krankheitsbegründungen besonders deutlich machen läßt.

Es fällt auf, daß in der pädiatrischen und kinderpsychiatrischen Forschung ein deutlich gesteigertes Interesse an hysterischen Phänomenen zu einer später nicht mehr zu beobachtenden Fülle von Veröffentlichungen über diese Thematik führte (4). Dies scheint u. a. dadurch erklärbar, daß Hysterie ein bei Kindern neues und im Gegensatz zur «Hysterie der Frau» noch nicht durch spekulative Theorien belastetes Krankheitsbild war und deshalb für neue Untersuchungen und Erklärungsweisen in besonderem Maße geeignet erschien. «Gerade durch die Beobachtung an Kindern ...», deren «Seele ... durchsichtiger ist, als die des Erwachsenen ...», seien «einige 
Aufschlüsse ... über das Wesen ... der Hysterie, bei der wir es ganz zweifellos mit einer psychischen Krankheit zu tun haben, zu erwarten» (30; S.841); so charakterisiert Friedrich Jolly (1844-1904) 1892 das Bemühen um eine kinderpsychiatrische Hysterieforschung, die infolge der Unbrauchbarkeit von funktionell-nervösen Erklärungsversuchen nun mehr die psychische Seite der Hysteriegenese aufgriff. Auch «der Einfluß einer psychischen Behandlung tritt hier viel deutlicher zu Tage als bei Erwachsenen». «So trägt denn Alles bei, diese Fälle für das Studium der Hysterie sehr geeignet zu machen ... m meint Marc Duvoisin 1889 (16; S. 260). Fast alle Autoren, die sich in der zweiten Hälfte des 19. Jahrhunderts und um die Jahrhundertwende mit der kindlichen Hysterie befaßten, berufen sich auf die durch umfangreiche statistische Erhebungen belegten Forschungsergebnisse in Paul Briquets «Traité de l'hystérie». «Mit der wichtigen Arbeit von Briquet, 1859, tritt die Frage in den Rahmen der modernen Pathologie ein. Das Vorkommen der Hysterie beim Kinde bedarf nach ihm keiner Discussion» schreibt Paul Bézy noch 1902 (4; S. 13). Das eigentliche Verdienst Briquets scheint aber darin zu liegen, daß er in einer Zeit, in der ein neurotisches Symptom ohne eine vorausgegangene funktionelle Störung im Gehirn oder Nervensystem ätiologisch nicht denkbar schien, die Frage nach der Bedeutung der hysterischen Symptome - basierend auf der klinischen Untersuchung und der persönlichen Lebensgeschichte der Kranken - ohne die Vorbehalte überkommener Theorien neu stellte. Das hysterische «Symptom» war für Briquet nicht mehr Ausdruck einer Funktionsstörung im Gehirn oder Nervensystem, sondern viel eher Ausdruck affektiver Erlebnisse auf dem Boden einer gesteigerten psychischen Beeindruckbarkeit. Die beobachtbare funktionell-nervöse Störung war im Konzept Briquets eindeutig sekundäre Folge der psychischen Störung und nicht umgekehrt (8; S. 3, 4). Eine Prädisposition für die Hysterie sah er in einer von der Kindheit an vorhandenen und beim weiblichen Geschlecht in besonderem Maße ausgeprägten $(8 ; \mathrm{S} .161)$ psychischen Beeindruckbarkeit sowie in der Wirkung affektiver Erlebnisse. Er erkannte das Kindesalter als den für die Ausbildung einer hysterischen Persönlichkeit wichtigsten Lebensabschnitt sowohl von der individuell verschiedenen Fähigkeit zur Aufnahme und Verarbeitung des gesamten psychischen Erlebens her als auch in bezug auf die Einwirkung affektiver Erlebnisse. Der Beginn der Hysterie sei jenseits des zwölften bis fünfzehnten Lebensjahres selten (8; S.161). Da Briquets Forschungen mehr aus einem praktisch-klinischen Bedürfnis als aus wissenschaftlichen Interessen hervorgingen (8; «Préface», V), wurde die Diskre- 
panz zwischen dem noch gültigen organischen Neurosekonzept und seiner klinisch-psychologischen Betrachtungsweise zunächst noch nicht zum Gegenstand wissenschaftlicher Auseinandersetzungen. Indem er die Hysterie als «névrose de l'encéphale» bezeichnete $(8 ; \mathrm{S} .3)$ und für die «impressionnabilité» der Hysterischen ein «élément affectif» im Nervensystem verantwortlich machte (8; S.161), blieb Briquet offiziell letztlich auch im Rahmen pathologisch-anatomischer Denkweisen. Sein Verdienst um die Geschichte der kindlichen Hysterie liegt nicht in der Konzeption einer neuen Hysterietheorie, sondern darin, daß er auf den Sinnzusammenhang zwischen den hysterischen Symptomen und der persönlichen Lebensgeschichte, insbesondere der Kindheit seiner Patienten hinwies.

Erst nachdem Jean-Martin Charcot (1825-1893) 1870 die Hysterie zum Hauptgegenstand seiner Forschungen gemacht hatte, wurde die bei Briquet $(11 ;$ S. 12) schon vorhandene, aber noch unausgesprochene Diskrepanz zwischen den «Principien ... der Pathologie», die «auch für die Neurosen Geltung haben», und den aus der «klinischen Beobachtung» (11; 13) gewonnenen Einsichten in den psychopathologischen «Mechanismus» gewisser hysterischer Phänomene (35; S. 164) zum neu diskutierten Problem. Charcot hielt die Hysterie für eine Gehirnneurose und betrachtete sie im Sinne der physiologisch-funktionellen Neurosetheorie als eine Erkrankung des Nervensystems «sine materia» (11; S.13). Durch die Anwendung von Hypnose, in der er eine experimentelle Methode zur Erforschung der Hysterie sah, glaubte er die von ihm so genannte «grande hystérie» oder «hystéro-épilepsie» als eine Krankheit mit konstanter Symptomatik und regelhaftem Verlauf erkannt zu haben (11; S.12). Seine diesbezüglichen Überlegungen wurden später als durch die Anwendung hypnotischer und suggestiver Verfahren entstandene «Züchtungsergebnisse» zurückgewiesen. Die Ätiologie und Pathogenese der Hysterie jedoch konnte Charcot nicht gemäß seinen ursprünglichen wissenschaftlichen Erwartungen verifizieren. Seine Forschungen in dieser Richtung blieben weitgehend erfolglos (21; S.40). Deshalb konnte er sich bei der klinischen Untersuchung seiner Patienten, insbesondere bei der Anwendung der Hypnose, der Erkenntnis bestimmter psychologischer Zusammenhänge der hysterischen Symptombildung nicht vollständig verschließen. Die psychologische Betrachtungsweise war für Charcot, der sich ebenso wie später Sigmund Freud (1856-1939) als Vertreter einer neuropathologisch-naturwissenschaftlichen Forschungsrichtung sah, jedoch eine außermedizinische Erklärung, die als echte Alternative für eine medizinisch-naturwissenschaftliche Patho-Ge- 
nese nicht in Frage kam. In zwei Bereichen erkannte er die Psychogenie schließlich doch als andersartigen methodischen Zugang zur Erklärung der hysterischen Symptome an: erstens bei den traumatischen Neurosen, die er als «traumatische Hysterien» bezeichnete (Fischer-Homberger 1970 b), zweitens bei der kindlichen Hysterie, insbesondere der Hysterie bei Knaben: «Es war um das Jahr 1880, als der berühmte Gelehrte der Salpêtrière durch klare Bestimmung der Grenzen, der Formen und der Behandlung der Hysterie das Vorkommen dieser Neurose bei Kindern anerkannte und dieselbe nicht nur in ihren mit Krämpfen einhergehenden, sondern auch in ihren larvirten schwieriger zu entdeckenden Formen zu erforschen unternahm. In der Folge traf man in seinen Vorlesungen alle Augenblicke Fälle von Kinderhysterie an, die den Gegenstand gründlicher Studien bildeten», schrieb Charcots Schüler Bézy 1902 (4; S. 15). Die Forschungen Charcots zur kindlichen Hysterie waren in zweifacher Hinsicht von ausschlaggebender Bedeutung: Erstens im Hinblick auf die Auswirkung für die kinderpsychiatrische Krankheitsforschung, zweitens wegen des außergewöhnlichen Stellenwertes, den Charcot insbesondere der kindlichen Hysterie gibt: «Denn was Charcot und seine Schüler in seinem Namen in Dingen der Hysterie sagten, hatte damals schlechthin wissenschaftliche Geltung» (Homburger, 1926) (29; S.353).

Es fällt auf, daß Charcot bei seinen Untersuchungen von Kindern sehr unmittelbar und weitgehend ohne theoretische Vorbehalte vorging. Aus seinen Erfahrungen mit Therapien hysterischer Kinder kam er zu der Auffassung, daß die Hysterie eine «Krankheit» sei, «bei welcher ... das psychische Element unstreitig in den meisten Fällen eine sehr beachtenswerthe, wenn nicht die Hauptrolle spielt» (11; S. 193). Er sagte weiterhin: «Es ist unbestreitbar, dass alles, was das Gemüth lebhaft ergreift und die Einbildungskraft mächtig anregt, das Auftreten der Hysterie bei dazu beanlagten Individuen in ganz besonderer Weise fördert» $(11 ; \mathrm{S} .182)$. Insoweit als er psychische «Einflüsse, die man als Traumen für die normale Geistesthätigkeit bezeichnen kann ...» für die Genese der kindlichen Hysterie verantwortlich machte, übernahm Charcot den Läsionsbegriff Trauma für die Psychogenese psychischer Krankheit (11; S. 182). In ähnlicher Weise wie die traumatische Hysterie erhielt damit auch die kindliche Hysterie, insbesondere die bei Knaben, eine wichtige Funktion in der Ätiologiediskussion über somatogene oder psychogene Entstehung psychischer Störungen. Charcot bewertete den pathogenen Einfluß von Lebensweise und Familienverhältnissen mindestens ebenso hoch wie eine nervöse Prädisposition $(11 ; \mathrm{S} .183,184)$ und 
machte die besondere Suggestibilität der Kinder und ihre Tendenz zur Nachahmung als Ursache für eine gegenseitige «psychische Ansteckung» verantwortlich (11; S. 182-189 und 190-201). Der Wert einer «moralischen oder psychischen Seite der Behandlung» wurde deshalb von ihm höher als andere spezifische therapeutische Maßnahmen (11; S. 191-194) eingeschätzt.

Der 21. Februar 1888, an dem Charcot in seiner Dienstagsvorlesung einen vierzehnjährigen Knaben mit großen hysterischen Anfällen vorstellte (12; S.133-141), wurde bei vielen Autoren als «ein klassischer Tag in der Geschichte der Klinik der Kinderhysterie» gewürdigt (29; S. 353; 4; S. 15). Dort nämlich sei zum erstenmal ausgesprochen worden, «dass man dieses Leiden für das nehmen muss, was es ist: Für eine psychische Erkrankung par excellence», schrieb Bézy 1902 (4; S. 16).

Insoweit als Charcot anhand von Fallbeispielen kindlicher Hysterie die Lebensgeschichte des jeweiligen Patienten und die die hysterischen Symptome auslösenden Erlebnisse in seine anamnestischen Erhebungen miteinbezog und aus dem Erfolg der «psychischen» Therapie Rückschlüsse auf die Psychogenese der Hysterie zog, konnte die Krankheit «kindliche Hysterie» - zunächst noch im Rahmen des organischen Neurosekonzeptes - als eine möglicherweise auch psychogen verursachte nervöse Funktionsstörung angesehen werden. Seit Charcot wurde die Vorstellung von der Psychogenie der Hysterie zum wissenschaftlichen Problem. Die kindliche Hysterie erhielt gerade durch den raschen Wechsel der Begründungen ihrer vermuteten Ätiologie - von der Somatogenese zur Psychogenese - eine frühe Eigenständigkeit als kinderpsychiatrische Diagnose in Abgrenzung von der Erwachsenen-Hysterie. Sie wurde auf der Basis des psychologischen Verständnisses des 19. Jahrhunderts zur ersten Kinderneurose, deren Psychogenese seither unbestritten ist. 
Zusammenfassung. Die historische Tradition, Hysterie als Krankheit des geschlechtsreifen Alters der Frau anzusehen, die in der Auffassung der Hysterie als Genitalneurose bis in das 19. Jahrhundert erhalten blieb, stand der Entwicklung eines kindlichen Hysteriebegriffes über mehr als zwei Jahrtausende entgegen. Dies verhinderte in der zweiten Hälfte des 19. Jahrhunderts, als sich aus der Pädiatrie und der Psychiatrie eine eigene Kinderpsychiatrie herausentwickelte - im Gegensatz zu zahlreichen anderen psychischen Störungen - eine wenig differenzierende Übertragung des Hysteriebegriffes der Erwachsenenpsychiatrie auf das Kindesalter und führte zu einer frühen Eigenständigkeit in der Betrachtung kindlicher hysterischer Zustände. Als neues und noch nicht durch spekulative Theorien belastetes Krankheitsbild erwies sich die kindliche Hysterie als geeignetes Forschungsobjekt vor allem psychologischer Betrachtungsweisen. Die kindliche Hysterie wurde damit zur ersten Kinderneurose, deren psychogene Genese im Sinne von Charcot und Freud unbestritten war.

\section{Literatur}

1. Ackerknecht, Erwin H., La Médecine à Paris entre 1800 et 1850. Les Conférences du Palais de la Découverte. Série D, No58. Paris 1958.

2. Aretäus von Kappadozien, Von den Ursachen und Kennzeichen rascher und langwieriger Krankheiten. Vier Bücher. Aus dem Griechischen mit beygefügten Anmerkungen übersetzt von F. D. Dewez, Wien 1790.

3. Baumès, Jean-Baptiste-Timothée, Von den Convulsionen der Kinder, von ihren Ursachen und ihrer Behandlung. Aus dem Französischen, Leipzig 1791.

4. Bézy, Paul, Die Hysterie im kindlichen und jugendlichen Alter, unter Mitwirkung von V.Bibent. Autorisierte deutsche Übersetzung von H. Brodtmann, Berlin 1902.

5. Binder, Hans, Der psychopathologische Begriff der Neurose. Schweiz. Arch. Neurol.89 (1962), S. 185-198.

6. Bouchut, Eugène, Handbuch der Kinderkrankheiten nebst einer Abhandlung über Diätetik und physische Erziehung. Nach der 2. französ. Originalaufl. dtsch. bearb. von B. Bischoff. Mit einem Vorwort von Fr. Rinecker, Würzburg 1854.

7. Brachet, Jean-Louis, Ueber die Convulsionen im kindlichen Alter. 2.vermehrte Aufl., aus dem Französ. von R. Finckh, Reutlingen 1838.

8. Briquet, Paul, Traité clinique et thérapeutique de l'hystérie, Paris 1859.

9. Cesbron, Henri, Histoire critique de l'hystérie. Thèse, Paris 1909.

10. Charcot, Jean-Martin, De l'hystérie chez les jeunes garçons, Leçon recueillie par Ch. Féré. Progr.méd.10 (1882), S.984-987, 1003-1004.

11. Charcot, Jean-Martin, Neue Vorlesungen über die Krankheiten des Nervensystems insbesondere über Hysterie. Autorisierte deutsche Ausgabe von Sigm. Freud, Leipzig und Wien 1886. 
12. Charcot, Jean-Martin, Leçons du Mardi à la Salpêtrière. Policlinique 1887-1888-1889. Notes de Cours de MM. Blin, Charcot et H. Colin (= Publications du Progrès Médical), 2 Bde., Paris 1889 und 1892.

13. Clopatt, Arthur, Etudes sur l'hystérie infantile. Thèse, Helsingfors 1888.

14. Cullen, William, Kurzer Inbegriff der medicinischen Nosologie: oder systematische Eintheilung der Krankheiten von Cullen, Linné, Sauvages, Vogel und Sagar. Nach der 3. Ausgabe, 2 Bde., Leipzig 1786. - 1. Ausgabe 1778.

15. Cullen, William, Anfangsgründe der praktischen Arzneykunst. 2 Teile in 1 Bd., Leipzig 1778-1780. Dritte Ausgabe, 3 Bde., Leipzig 1800.

16. Duvoisin, Marc, Über infantile Hysterie. Jb. Kinderhk. N.F. 29 (1889), S.287-332.

17. Emminghaus, Hermann, Die psychischen Störungen des Kindesalters. In: Handbuch der Kinderkrankheiten, hrsg. von C. Gerhardt. Nachtrag II, Tübingen 1887, 1-293.

18. Faye, Ludvig, Hysteriforme und damit verwandte nervöse Erscheinungen, in: Beitrag zur Kenntnis von Nervenkrankheiten bei Kindern. Schmidts Jb. ges. Med. 176 (1877), S. 156.

19. Fischer-Homberger, Esther, Hysterie und Misogynie - ein Aspekt der Hysteriegeschichte. Gesnerus 26 (1969), S.117-127.

20. Fischer-Homberger, Esther, Hypochondrie. Melancholie bis Neurose. Krankheiten und Zustandsbilder. Bern-Stuttgart-Wien 1970 a.

21. Fischer-Homberger, Esther, Charcot und die Ätiologie der Neurosen. Gesnerus 27 (1970 b), S. 35-46.

22. Frank, Johann-Peter, Grundsätze über die Behandlung der Krankheiten des Menschen, zu akademischen Vorlesungen bestimmt, 9 Bde., Mannheim und Leipzig 1829-1831.

23. Galenus, Claudius, Opera omnia. Griechisch und lateinisch hrsg. von Carolus Gottlob Kühn, 20 Bde., Leipzig 1821-1833.

24. Georget, Etienne-Jean, De la physiologie du système nerveux, et spécialement du cerveau. Recherches sur les maladies nerveuses en général, et en particulier sur le siège, la nature et le traitement de l'hystérie, de l'hypochondrie, de l'épilepsie et de l'asthme convulsif. 2 Bde., Paris 1821.

25. Gerhardt, Carl, Lehrbuch der Kinderkrankheiten, Tübingen 1861.

26. Henoch, Eduard, Die hysterischen Affektionen der Kinder. Wien. med. Presse XXII (1881), S.916-918, 951-952, 980-982, 1006-1009.

27. Hippokrates, Die Werke des Hippokrates. Die hippokratische Schriftensammlung in neuer deutscher Übersetzung. Hrsg. von Richard Kapferer unter Mitwirkung von Georg Sticker u. a., 25 Teile und 1 Erg. T., Stuttgart 1934-1939.

28. Hippokrates, Über die Umwelt (De aere aquis locis), griech. und deutsch, hrsg. und übers. von Hans Diller, Berlin 1970.

29. Homburger, August, Vorlesungen über Psychopathologie des Kindesalters. Sonderausgabe der wissenschaftlichen Buchgesellschaft, Darmstadt 1972, unveränderter reprografischer Nachdruck der Ausgabe Berlin 1926.

30. Jolly, Friedrich, Über Hysterie bei Kindern. Berliner klin. Wschr. XXIX (1892), S. 841-845.

31. Jörg, Johann Christian Gottfried, Handbuch zum Erkennen und Heilen der Kinderkrankheiten, Leipzig 1826.

32. Kaech, René, Die somatische Auffassung der Hysterie. In: Ciba Zschr. 47 (1950), S. 1558-1568. 
33. Kennedy, Henry, Einige neuere Beobachtungen über die Paralyse der Kinder. J. Kinderkrkh. 34 (1862), S.379-385.

34. Kindt, Hildburg, Vorstufen der Entwicklung zur Kinderpsychiatrie im 19. Jahrhundert. Zur Wertung von Hermann Emminghaus und seiner «Psychischen Störungen des Kindesalters» (1887) (= Freiburger Forschungen zur Medizingeschichte. Neue Folge, hrsg. von E. Seidler, G. Keil und B. Kuhlo, Bd. 1), Freiburg i. Br. 1971.

35. Kloë, Elisabeth, Hysterie im Kindesalter. Zur Entwicklung des kindlichen Hysteriebegriffes (= Freiburger Forschungen zur Medizingeschichte. Neue Folge, hrsg. von E. Seidler und B. Kuhlo, Bd.9), Freiburg i. Br. 1979.

36. Kraepelin, Emil, Psychiatrie. Ein kurzes Lehrbuch für Studirende und Ärzte. 3.Aufl., Leipzig 1889.

37. Landouzy, Marc-Hector, Traité complet de l'hystérie, Paris-London 1846.

38. Lange, Carl, in: Beitrag zur Kenntniss von Nervenkrankheiten bei Kindern. Schmidts Jb. ges. Med. 176 (1877), S.156-159.

39. Lieutaud, Joseph, Précis de la médecine pratique. $4^{\mathrm{e}}$ éd., revue et augmentée par l'auteur. 3 Bde., Paris 1776.

40. Lopez Piñero, José M., Origenes históricos del concepto de neurosis (= Cuadernos Valencianos de historia de la medicina I). Valenzia 1963.

41. Louyer-Villermay, Jean-Baptiste, Hystérie. In: Dictionnaire des sciences médicales, Bd.23, Paris 1818, S.226-272.

42. Pinel, Philippe, Nosographie philosophique, ou la méthode de l'analyse appliquée à la médecine. 6. Aufl., 3 Bde., Paris 1818.

43. Piso, Carolus (= Lepois, Charles), Selectiorum observationum et consiliorum liber singularis, Leiden 1714.

44. Platon, Timaios oder die Schrift über die Natur. Übers. und erläutert von Richard Kapferer in Zusammenarbeit mit Anton Fingerle, Stuttgart 1952.

45. Raulin, Joseph, Traité des affections vaporeuses du sexe avec l'exposition de leurs symptômes, de leurs différentes causes et la méthode de les guérir, Paris 1758.

46. Romberg, Moritz Heinrich, Lehrbuch der Nerven-Krankheiten des Menschen, 2 Bde., 3. veränderte Aufl., Berlin 1853.

47. Rothschuh, Karl Eduard, Vom Spiritus animalis zum Nervenaktionsstrom. Ciba Zschr. 89 (1958), S. 2950-2980.

48. Seeligmüller, Otto, Ueber Chorea magna und ihre Behandlung. Dtsch. med. Wschr. 7 (1881), S.584.

49. Seidler, Eduard, Kindliche Anfallsleiden in der pädiatrischen Literatur des 18. Jahrhunderts. Mschr. Kinderhk. 112 (1964), S.393-398.

50. Smidt, Hermann, Über das Vorkommen der Hysterie bei Kindern. Jb. Kinderhk., N.F. 15 (1880), S. 1-22.

51. Soranus von Ephesos, Soranus' Gynecology. Translated and with an Introduction by Owsei Temkin. Baltimore 1956.

52. Sydenham, Thomas, The Works of Thomas Sydenham, M.D. Translated from the Latin edition of Dr.Greenhill with a «Life of the Author» by R.G.Latham, 2 Bde., London 1848-1850.

53. Veith, Ilza, Hysteria: The history of a disease, Chicago und London 1965.

54. Willis, Thomas, Pathologiae cerebri et nervosi generis specimen. In quo agitur de morbis convulsivis, et de scorbuto. Amsterdam 1668. 


\section{Summary}

Over a certain period hysteria was regarded as a disease of the sexually mature woman. This conception was not applicable on children's hysteria.

The observation of hysteria in childhood calls upon a peculiar way of consideration, which is described in detail. The children's hysteria is regarded as the first definable neurosis of children with uncontested psychogenesis.

Dr.med. Elisabeth Klö̈

Psychiatrische Universitätsklinik

Voßstraße 4

D-6900 Heidelberg
Prof. Dr. med. Hildburg Kindt

Psychiatrische und Neurologische Universitätsklinik Hauptstraße 5

D-7800 Freiburg i. Br. 